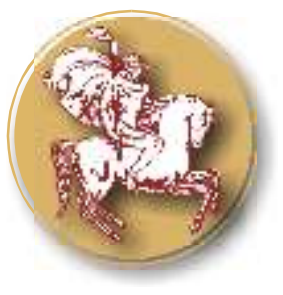

Trakia Journal of Sciences, No 2, pp 185-187, 2021

Copyright (C) 2021 Trakia University

Available online at:

http://www.uni-sz.bg

ISSN 1313-3551 (online)

doi:10.15547/tjs.2021.02.012

Review

\title{
COVID-19 AND BRONCHIAL ASTHMA
}

\author{
D. Dimov ${ }^{1}$, T. Atanasov ${ }^{2}$ \\ ${ }^{1}$ Department of Medical Chemistry and Biochemistry, Medical Faculty, Trakia University, \\ Stara Zagora, Bulgaria \\ ${ }^{2}$ General Hospital for Active Treatment "St. Ekaterina" Dimitrovgrad, Bulgaria
}

\begin{abstract}
Severe coronavirus disease-19 (COVID-19) presents with progressive dyspnea, resulting from acute lung inflammatory edema leading to hypoxia. Asthma has been cited as a potential risk factor for severe COVID-19 like other diseases that affect the respiratory tract. However, so far conflicting results have been published over the last few months and there is still no prove that there is a putative association between these two diseases. In the current mini-review we attempt to summarize the available information from the scientific literature concerning the relation of Bronchial asthma and the severity and clinical course of COVID-19 disease.
\end{abstract}

Key words: COVID-19, SARS-CoV-2, Bronchial asthma

\section{Clinical symptoms in COVID-19 infection}

Coronaviruses are the largest known positivesense RNA viruses, with a wide range of hosts, and have serious negative effects on human and animal health. Coronaviruses known to infect humans, include low-pathogenic Coronaviruses (CoV-229E, CoV-NL63, CoVOC43, and CoV-HKU1) causing mild to moderate illness and high-pathogenic coronaviruses that can lead to severe, potentially deadly diseases $(1,2)$. In the twentyfirst century, outbreaks with severe acute respiratory syndrome coronavirus (SARS$\mathrm{CoV}$ ) and Middle East respiratory syndrome coronavirus (MERS-CoV) have caused massive impacts on public health as well as socioeconomic aspects. Notably, the pandemic of coronavirus disease 2019 (COVID-19) caused by the novel Coronaviruse - Severe Acute Respiratory Syndrome CoronaVirus 2 (SARS-CoV-2) has posed a serious threat to global health since December $2019(1,2)$.

It has been believed that SARS-CoV-2 spreads predominantly by droplets, aerosols, and contact transmission and can also be detected in stool, urine, and blood $(1,2)$. The majority

\footnotetext{
*Correspondence to: Dimo Dimov, Dept. Medical Chemistry and Biochemistry, Medical Faculty, Trakia University, Stara Zagora,

E-mail:dmdimov65@yahoo.com
}

of infected people have mild or no symptoms, but a proportion of patients display severe and rapid progression of the disease, leading to acute lung injury/acute respiratory distress syndrome and/or multiple organ failure $(3,4)$. The median incubation period is estimated to be 5.1 days, with $97.5 \%$ of symptomatic infections becoming evident within 11.5 days (5). The most common symptoms include fever $(88.5 \%)$, cough (68.6\%), myalgia or fatigue $(35.8 \%)$, expectoration $(28.2 \%)$, and dyspnea (21.9\%) (6). Laboratory examinations have shown that severe COVID-19 patients have decreased numbers of neutrophils, lymphocytes, and eosinophils and neutrophilto-lymphocyte ratios, suggesting that abnormal, over activated immunity might be a possible mechanism (7).

\section{Association of Bronchial asthma and COVID-19 infectious}

In Bronchial asthma $80 \%$ of exacerbations are caused by respiratory viruses in children and half of such in adults. In response to respiratory viruses, people with asthma have increased clinical severity, bronchial hyperreactivity, impaired lung function and eosinophilic inflammation related to augmented Th2 or impaired Th1 or IL-10 responses (8) 
It is important to know that so far there is no evidence of increased infection rates in those patients with asthma. The Centers for Disease Control and Prevention states that patients with moderate-severe asthma could be at greater risk for more severe disease, however so far there are no published data that supports this notion. There have been many studies exploring the relationship between COVID-19 and asthma (9-11). So far the majority of these studies have not found increased risk of COVID-19 disease severity in people with asthma. Also, there appears to be no indication that asthma is a risk factor for developing COVID-19 disease.

Nevertheless, some studies have suggested that non-allergic asthma may be associated with more severe COVID-19 disease (9). However, it is not clear in these studies whether subjects did not have chronic obstructive pulmonary disease (COPD), which is a well-established risk for severe COVID-19. It appears that there is either no risk or at most a very slight risk for more severe COVID-19 disease in non-allergic asthma patients compared to other risk factors like COPD, obesity, etc., that have consistently been linked to more severe COVID-19 disease (9).

Bearing in mind that asthma patients have impaired immune response against virus infections and they can trigger an exacerbation of asthma, the management of these patients becomes more complicated during the COVID-19 pandemic (9). In theory, it seems that pre-existing asthma should potentially have an influence on SARS-CoV-2 susceptibility and disease control. However, existing studies are not showing a high percentage of asthma in COVID -19 patients (9). Few studies have listed asthma as comorbidity, which is insufficient to support any conclusions. The China Center for disease control posted that only $2.4 \%$ of 44,672 patients were with chronic respiratory disease including asthma (9). In Israel a study of 37,469 subjects tested for COVID-19 RT-PCR showed that only 153 subject who were positive had bronchial asthma (6.75\%) (9). These results are showing a lower COVID-19 susceptibility in patients with preexisting asthma (9). A study from Lombardy, Italy, has also shown relatively low prevalence of asthma (10). A study in Korea done by Choi at al. included 7590 COVID -19 patient population,
DIMOV D., et al.

found a higher frequency of asthma compared to the general population (2.9 versus $1.6-2.2 \%$ ) (11). In a New York City, USA study carried on by Richardson and et al. among 5700 patients show that 9\% were COVID-19 positive with asthma. These results, so far, have shown that asthma is not a predisposing or aggravating factor for COVID-19 (12). Some cytokines which are prevalent in asthma -IL-9, IL-4, IL-13 have anti-inflammatory effects (13): IL-9 reduces the TNF- $\alpha$ and IL10 secretion; IL-4 suppresses the development of Th1 cells from Th0; IL-13 has an immunoregulatory effect by inhibiting the secretion of IL- $1 \alpha$ and $-1 \beta$, IL- 6 and TNF- $\alpha$ $(14,15)$. Therefore the effect of the type 2 cytokines might counteract the accumulation of pro-inflammatory cytokines during the pathogenesis of COVID-19.

The expression of the ACE2 receptor (the entry receptor for SARS-CoV-2) might be affected in asthma patients. A study done by Jackson and al. examining the RNA-seq data in airway cells from 3 different cohorts reported that respiratory allergy and controlled allergen exposures are associated with significant reductions in ACE2 expression (16). There is still no solid proof that a distinct type 2 immune response contributes to COVID-19.

\section{CONCLUSION}

Despite the impaired antiviral immune responses and the tendency for acute exacerbation, there is little evidence showing that asthma patients have increased susceptibility or severity of SARS-CoV-2 infection than others. It is very interesting to investigate whether features of the disease like type 2 immune response, asthma therapeutics, or all of them are able to provide some protective effects against COVID-19. Further studies are needed to explore the relationship between COVID-19 and asthma and other allergic diseases.

\section{ACKNOWLEDGMENTS}

This paper was supported by a project 6/2021, MF, Trakia University, Stara Zagora.

\section{REFERENCES}

1. Wang W, Xu Y, Gao R, Lu R, Han K, Wu G, Tan W. Detection of SARS-CoV-2 in Different Types of Clinical Specimens. JAMA. 323(18):1843-1844, 2020.

2. Peng L, Liu J, Xu W, Luo Q, Chen D, Lei Z, Huang Z, Li X, Deng K, Lin B, Gao Z. 
SARS-CoV-2 can be detected in urine, blood, anal swabs, and oropharyngeal swabs specimens. J Med Virol. 92(9):16761680, 2020.

3. Huang C, Wang Y, Li X, Ren L, Zhao J, Hu Y, Zhang L, Fan G, Xu J, Gu X, Cheng Z, Yu T, Xia J, Wei Y, Wu W, Xie X, Yin W, Li H, Liu M, Xiao Y, Gao H, Guo L, Xie J, Wang G, Jiang R, Gao Z, Jin Q, Wang J, Cao B. Clinical features of patients infected with 2019 novel coronavirus in Wuhan, China. Lancet. 395(10223):497-506, 2020.

4. Chen N, Zhou M, Dong X, Qu J, Gong F, Han Y, Qiu Y, Wang J, Liu Y, Wei Y, Xia $\mathrm{J}, \quad \mathrm{Yu} \mathrm{T}$, Zhang $\mathrm{X}$, Zhang $\mathrm{L}$. Epidemiological and clinical characteristics of 99 cases of 2019 novel coronavirus pneumonia in Wuhan, China: a descriptive study. Lancet. 395(10223):507-513, 2020.

5. Lauer SA, Grantz KH, Bi Q, Jones FK, Zheng Q, Meredith HR, Azman AS, Reich NG, Lessler J. The Incubation Period of Coronavirus Disease 2019 (COVID-19) From Publicly Reported Confirmed Cases: Estimation and Application. Ann Intern Med. 172(9):577-582, 2020.

6. Liu S, Zhi Y, Ying S. COVID-19 and Asthma: Reflection during the Pandemic. Clin Rev Allergy Immunol. 59(1):78-88, 2020.

7. https://www.aaaai.org/conditions-andtreatments/asthma

8. WHO. COVID-19 situation reports. https://www.who.int/emergencies/d iseases/novel-coronavirus-2019/situationreports (2020).

9. Green I, Merzon E, Vinker S, Golan-Cohen A, Magen E. COVID-19 Susceptibility in Bronchial Asthma. J Allergy Clin Immunol Pract:;9(2):684-692, 2021.

10.Grasselli G, Zangrillo A, Zanella A, Antonelli M, Cabrini L, Castelli A, Cereda D, Coluccello A, Foti G, Fumagalli R, Iotti G, Latronico N, Lorini L, Merler S, Natalini G, Piatti A, Ranieri MV, Scandroglio AM, Storti E, Cecconi M, Pesenti A; COVID-19 Lombardy ICU Network. Baseline
DIMOV D., et al.

Characteristics and Outcomes of 1591 Patients Infected With SARS-CoV-2 Admitted to ICUs of the Lombardy Region, Italy. JAMA. 323(16):1574-1581, 2020.

11. Choi HG, Wee JH, Kim SY, Kim JH, Il Kim H, Park JY, Park S, Il Hwang Y, Jang SH, Jung KS. Association between asthma and clinical mortality/morbidity in COVID19 patients using clinical epidemiologic data from Korean Disease Control and Prevention. Allergy. 76(3):921-924, 2021.

12.https://link.springer.com/article/10.1007/s1 2016-020-08797-3\#ref-CR44u

13. Richardson S, Hirsch JS, Narasimhan M, Crawford JM, McGinn T, Davidson KW; the Northwell COVID-19 Research Consortium, Barnaby DP, Becker LB, Chelico JD, Cohen SL, Cookingham J, Coppa K, Diefenbach MA, Dominello AJ, Duer-Hefele J, Falzon L, Gitlin J, Hajizadeh N, Harvin TG, Hirschwerk DA, Kim EJ, Kozel ZM, Marrast LM, Mogavero JN, Osorio GA, Qiu M, Zanos TP. Presenting Characteristics, Comorbidities, and Outcomes Among 5700 Patients Hospitalized With COVID-19 in the New York City Area. JAMA. 323(20):20522059, 2020.

14.Dembic Z Chapter 6 - cytokines of the immune system: interleukins. In: Dembic $Z$ (ed) The cytokines of the immune system. Academic Press, Amsterdam, pp143-239. 2015.

15.de Vries JE. The role of IL-13 and its receptor in allergy and inflammatory responses. $J$ Allergy Clin Immunol. 102(2):165-169, 1998.

16.Jackson DJ, Busse WW, Bacharier LB, Kattan M, O'Connor GT, Wood RA, Visness CM, Durham SR, Larson D, Esnault S, Ober C, Gergen PJ, Becker P, Togias A, Gern JE, Altman MC. Association of respiratory allergy, asthma, and expression of the SARS-CoV-2 receptor ACE2. J Allergy Clin Immunol. 146(1):203-206.e3, 2020. 